\title{
ANDERSON T-MOTIVES AND ABELIAN VARIETIES WITH MIQF: RESULTS COMING FROM AN ANALOGY
}

\author{
A. GrishKov, D. LOGACHEV ${ }^{1}$
}

\begin{abstract}
Analogy between Anderson T-motives and abelian varieties with multiplication by an imaginary quadratic field (MIQF) is a source of 2 results:

1. A description of abelian varieties with MIQF of dimension $r$ and signature $(n, r-n)$ in terms of "lattices" of dimension $r$ in $\mathbb{C}^{n}$;

2. A construction of exterior powers of abelian varieties with MIQF having $n=1$.
\end{abstract}

\section{Introduction.}

The origin of the present paper is an analogy 1.8 (known for experts) between the following two objects corresponding to the function field case and the number field case respectively:

A. A T-motive $M$ of rank $r$ and dimension $n$, pure, uniformizable, having the nilpotent operator $N$ equal to 0 (see Section 1 for the exact definitions and the origin of the analogy),

and

B. An abelian variety $A$ over $\mathbb{C}$ with multiplication by an imaginary quadratic field $K$ (abbreviated as an abelian variety with MIQF), of dimension $r$ and of signature $(n, r-n)$.

Using well-known constructions for T-motives, we get analogous constructions for abelian varieties with MIQF. Firstly, let us consider the lattice $L(M)$ associated to a T-motive $M$ of type $\mathbf{A}$ :

C. $L(M)$ is an $r$-dimensional $\mathbb{F}_{p}[\theta]$-lattice in $\mathbb{C}_{\infty}^{n}$, where $\mathbb{F}_{p}[\theta]$ (resp. $\left.\mathbb{C}_{\infty}\right)$ is the functional analog of $\mathbb{Z}$ (resp. $\mathbb{C}$ ).

Let us consider the lattice $L(A)$ associated to an abelian variety $A$ with MIQF:

1991 Mathematics Subject Classification. Primary 11G09, 11G10, 11G15, 14 K99.

Key words and phrases. Anderson T-motives; abelian varieties with multiplication by an imaginary quadratic field; exterior power of abelian varieties.

Thanks: Alain Genestier and Laurent Fargues attracted the attention of the authors to the main analogy of the present paper. The authors are grateful to FAPESP, São Paulo, Brazil for a financial support (process No. 2017/19777-6). The first author is grateful to SNPq, Brazil, to RFBR, Russia, grant 16-01-00577a (Secs. 1-4), and to Russian Science Foundation, project 1611-10002 (Secs. 5-8) for a financial support. The authors are grateful to an anonymous reviewer for some important remarks.

${ }^{1}$ E-mails: shuragri@gmail.com; logachev94@gmail.com (corresponding author) 
D. $L(A)$ is an $r$-dimensional $O_{K}$-lattice in $\mathbb{C}^{r}\left(L(A)\right.$ is not an $O_{K}$-submodule of $\mathbb{C}^{r}$ treated as $O_{K}$-module).

Objects of types (C) and (D) are not quite analogous, isn't it? The first result of the present paper is Theorem 2.6 which gives us - roughly speaking — that

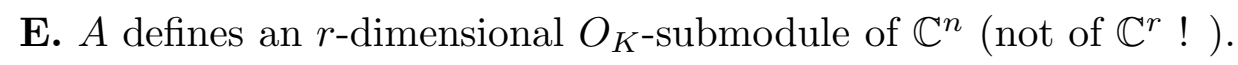

(E) is an analog of $(\mathbf{C})$.

Secondly, it is known that if $M$ of type $\mathbf{A}$ has $n=1$ then its $k$-th exterior power $\lambda^{k}(M)$ is also an object of type $\mathbf{A}$. By analogy, we can expect that if $A$ is an abelian variety with MIQF having $n=1$ then $\lambda^{k}(A)$ is defined, and is also an abelian variety with MIQF. We really give this construction (Section 3 ).

Both these results are of elementary nature, they could be known to Riemann. ${ }^{2}$ Probably this analogy will be a source of more results. For example, it would be interesting to define analogs of T-motives having $N \neq 0$.

The paper is organized as follows. In Section 1 we recall definitions of T-motives and we formulate the results which are starting points (using the analogy 1.8) of the results of Sections 2, 3. Section 2 contains the exact statement and the proof of (E). In Section 3 we apply this result to construct exterior powers of abelian varieties with MIQF having $n=1$. Formally, these sections are independent of Section 1, i.e. they do not require any knowledge of functional case.

Remark. Many constructions/results of the theory of Drinfeld modules are analogs of the corresponding constructions/results of the theory of abelian varieties. The direction of analogies of the present paper is the opposite: from Drinfeld modules to abelian varieties.

\section{Section 1. Origin of construction: T-motives.}

A standard reference for T-motives is [G], Section 5, we shall use its notations if possible. Let $\mathfrak{r}$ be a power of a prime number, ${ }^{3} \mathbb{F}_{\mathfrak{r}}(\theta)$ (resp. $\mathbb{F}_{\mathfrak{r}}\left(\left(\theta^{-1}\right)\right)$ ) the functional analog of $\mathbb{Q}$ (resp. of $\mathbb{R})$, and $\mathbb{C}_{\infty}$ - the completion of the algebraic closure of $\mathbb{F}_{\mathfrak{r}}\left(\left(\theta^{-1}\right)\right)$ — the functional analog of $\mathbb{C}$.

The definition of a T-motive $M$ is given in [G], Definitions 5.4.2, 5.4.12 (Goss uses another terminology: "abelian T-motive" of $[\mathrm{G}]=$ "T-motive" of the present paper; $L$ of Goss should be considered as $\left.\mathbb{C}_{\infty}\right)$. Particularly, $M$ is a free $\mathbb{C}_{\infty}[T]$-module of dimension $r$ (this number $r$ is called the rank of $M$ ) endowed by a $\mathbb{C}_{\infty}$-skew-linear operator $\tau$ satisfying some properties. A nilpotent operator $N=N(M)$ associated to a T-motive is defined in $[\mathrm{G}]$, Remark 5.4.3.2. We shall consider only pure $([\mathrm{G}]$, Definition 5.5.2) uniformizable ([G], Theorem 5.9.14, (3)) T-motives. Its dimension $n$ is defined in $[\mathrm{G}]$, Remark 5.4.13.2 (Goss denotes the dimension by $\rho$ ). Condition $N=0$ implies $n \leq r$. A T-motive of dimension 1 is the same as a Drinfeld module, they are all pure, uniformizable, and their $N$ is 0 .

If $N(M)=0$ then attached to such T-motive is a lattice $L=L(M)$ which is a free $r$-dimensional $\mathbb{F}_{\mathfrak{r}}[\theta]$-module in $\mathbb{C}_{\infty}^{n}$, and if $N \neq 0$ then $L(M)$ is a slightly more

\footnotetext{
${ }^{2}$ Claire Voisin told me that she knew the construction of Section 3 (not published), but she did not know its relation with exterior power of Drinfeld modules.

${ }^{3}$ We use notation $\mathfrak{r}$ instead of $r$ of $[\mathrm{G}]$ in order do not confuse with the rank of a T-motive.
} 
complicated object, we do not need to consider details for this case. Inclusion of $L$ in $\mathbb{C}_{\infty}^{n}$ defines a surjective map

$$
\alpha=\alpha(M): L \underset{\mathbb{F}_{\mathfrak{r}}[\theta]}{\otimes} \mathbb{C}_{\infty} \rightarrow \mathbb{C}_{\infty}^{n}
$$

having the property

$$
\text { Restriction of } \alpha \text { to } L \underset{\mathbb{F}_{\mathfrak{r}}[\theta]}{\otimes} \mathbb{F}_{\mathfrak{r}}\left(\left(\theta^{-1}\right)\right) \text { is injective }
$$

Tensor product of T-motives $M_{1}, M_{2}$ is simply their tensor product over $\mathbb{C}_{\infty}[T]$, where the action of $\tau$ is defined by the formula $\tau\left(m_{1} \otimes m_{2}\right)=\tau\left(m_{1}\right) \otimes \tau\left(m_{2}\right)$. If both $M_{1}, M_{2}$ are pure uniformizable then $M_{1} \otimes M_{2}$ is pure uniformizable as well. The same definition holds for exterior (resp. symmetric) powers of $M$. The dual T-motive $M^{*}$ is defined in [GL07].

1.3. It is easy to check that even if $N\left(M_{1}\right), N\left(M_{2}\right)$ are 0 then $N\left(M_{1} \otimes M_{2}\right)$, $N\left(\lambda^{k}\left(M_{1}\right)\right), N\left(S^{k}\left(M_{1}\right)\right)$ are not 0 . The only exception: if $M$ is a Drinfeld module $(\Longleftrightarrow n=1)$ then $N\left(\lambda^{k}(M)\right)=0$, this is an elementary calculation.

There is a natural problem to describe $L\left(M_{1} \otimes M_{2}\right), L\left(\lambda^{k}\left(M_{1}\right)\right), L\left(S^{k}\left(M_{1}\right)\right)$ in terms of $L\left(M_{1}\right), L\left(M_{2}\right)$. It was solved by Anderson (non-published), a formula which is equivalent to this description is stated without proof in $[\mathrm{P}]$, end of page 3 . See [GL07], Section 6 for more details and for the proof of the formula of Pink in the case when $N\left(M_{1}\right), N\left(M_{2}\right)$ are 0 . Let us state the theorem for the case when all $N$ are 0 (and hence all $L$ are lattices), i.e. $M$ is a Drinfeld module of rank $r$. We need

Definition 1.4. For a short exact sequence of vector spaces over a field

$$
0 \rightarrow B_{1} \stackrel{i}{\rightarrow} B_{2} \rightarrow C \rightarrow 0
$$

we define its $k$-th exterior power as the following exact sequence:

$$
0 \rightarrow \lambda^{k}\left(B_{1}\right) \stackrel{\lambda^{k}(i)}{\rightarrow} \lambda^{k}\left(B_{2}\right) \rightarrow C_{k} \rightarrow 0
$$

Now let us consider the exact sequences for $M, \lambda^{k}(M)$

$$
\begin{gathered}
0 \rightarrow \operatorname{Ker} \alpha(M) \rightarrow L(M) \underset{\mathbb{F}_{\mathfrak{r}}[\theta]}{\otimes} \underset{\mathbb{C}_{\infty}}{\mathbb{\alpha}^{(M)}} \mathbb{C}_{\infty} \rightarrow 0 \\
0 \rightarrow \operatorname{Ker} \alpha\left(\lambda^{k}(M)\right) \rightarrow L\left(\lambda^{k}(M)\right) \underset{\mathbb{F}_{\mathfrak{r}}[\theta]}{\otimes} \mathbb{C}_{\infty} \stackrel{\alpha\left(\lambda^{k}(M)\right)}{\rightarrow} \mathbb{C}_{\infty}^{n(r, k)} \rightarrow 0
\end{gathered}
$$

where maps $\alpha(M), \alpha\left(\lambda^{k}(M)\right)$ are from 1.1, and $n(r, k)=\left(\begin{array}{c}r-1 \\ k-1\end{array}\right)$ is the dimension of $\lambda^{k}(M)$.

Theorem 1.7. There exists a canonical isomorphism from the $k$-th exterior power of (1.5) to (1.6) such that the image of $\lambda^{k}(L(M))$ in (1.5) is $L\left(\lambda^{k}(M)\right)$ in (1.6). 
Proof is completely analogous to the proofs for the case of dual T-motives ([GL07], Theorem 5) and for the case of tensor product of T-motives having $N=0$ ([GL07], Theorem 6.2), hence it is omitted.

1.8. Origin of the analogy. We give here only a sketch of definitions; see for example $[\mathrm{W}]$ for the exact statements. Let $X$ be a Shimura variety and $G$ a reductive group over $\mathbb{Q}$ associated to $X$ according Deligne. Let $p$ be a prime of good reduction of $X . \mathbb{H}_{p}(X)$ - the $p$-part of the Hecke algebra of $X$ - is isomorphic to $\mathcal{H}\left(G\left(\mathbb{Q}_{p}\right)\right)$ - the algebra of double cosets $G\left(\mathbb{Z}_{p}\right) g G\left(\mathbb{Z}_{p}\right), g \in G\left(\mathbb{Q}_{p}\right)$. There exists a Levi subgroup $M$ of $G$ having the following property:

The $p$-part of the Hecke algebra of $\tilde{X}$ (the reduction of $X$ at $p$ ) is isomorphic to $\mathcal{H}\left(M\left(\mathbb{Q}_{p}\right)\right)$.

See, for example, [W], p. 44, $\left(^{*}\right)$ and p. 49, (1.10) for the definition and properties of $M$.

Example. If $X$ is a Shimura variety parametrizing abelian varieties with multiplication by an imaginary quadratic field $K$, of dimension $r$ and of signature $(n, r-n)$, then $G(\mathbb{Q})=G U(n, r-n)(K)$. If $p$ splits in $K / \mathbb{Q}$ then $G\left(\mathbb{Q}_{p}\right)=G L_{r}\left(\mathbb{Q}_{p}\right) \times G L_{1}\left(\mathbb{Q}_{p}\right)$. Let $i_{n, r-n}: G L_{n}\left(\mathbb{Q}_{p}\right) \times G L_{r-n}\left(\mathbb{Q}_{p}\right) \hookrightarrow G L_{r}\left(\mathbb{Q}_{p}\right)$ be the inclusion of the subgroup of $(n \times r-n)$-block diagonal matrices. Then the inclusion $M\left(\mathbb{Q}_{p}\right) \hookrightarrow G\left(\mathbb{Q}_{p}\right)$ is the inclusion

$$
i_{n, r-n} \times G L_{1}\left(\mathbb{Q}_{p}\right): G L_{n}\left(\mathbb{Q}_{p}\right) \times G L_{r-n}\left(\mathbb{Q}_{p}\right) \times G L_{1}\left(\mathbb{Q}_{p}\right) \hookrightarrow G L_{r}\left(\mathbb{Q}_{p}\right) \times G L_{1}\left(\mathbb{Q}_{p}\right)
$$

For the function field case an analog of this theory is conjectural, but preliminary results of [L] show that for abelian Anderson T-motives of rank $r$ and dimension $n$ we have the same groups (up to the factor $G L_{1}$ ): $G=G L_{r}, M=G L_{n} \times G L_{r-n} \subset$ $G L_{r}$.

As a corollary we get that the dimensions of moduli spaces of both types of objects (T-motives; abelian varieties with MIQF) are equal: they are $n(r-n)$.

The below sections 2, 3 contain constructions of the number field case analogs of the map $\alpha$ of (1.1), and of Theorem 1.7 respectively. From one side, finding of these constructions was inspired by the analogy; from another side, their existence is a support to the analogy.

2. Abelian varieties with MIQF. We shall fix an imaginary quadratic field $K=\mathbb{Q}(\sqrt{-\Delta})$. For simplicity, an abelian variety $A$ is treated up to isogeny, and we restrict ourselves only by one fixed polarization form.

The main theorem 2.6 establishes an equivalence of objects of types $\mathbf{B}$ and $\mathbf{E}$.

Definitions for the type B. Let $A=V / D_{\mathbb{Z}}, V=\mathbb{C}^{r}$ be an abelian variety with MIQF, $L=D_{\mathbb{Z}} \otimes_{\mathbb{Z}} \mathbb{Q}$. Since we consider $A$ up to isogeny, we shall deal only with $L$ and not with $D_{\mathbb{Z}}$. We fix an inclusion $\iota: K \hookrightarrow$ End $(A) \otimes_{\mathbb{Z}} \mathbb{Q}$ defining multiplication, and we fix an Hermitian polarization form $H=B+i \Omega$ of $A$ on $V$, where $B$ and $\Omega$ are respectively its real and imaginary parts. There are two structures of $K$-module on $V$ : the ordinary one which is the restriction of the $\mathbb{C}$-module structure, and the *-structure (multiplication is denoted by $k * v, k \in K, v \in V$ ) coming from $\iota . L$ is a $K$-*-module. We choose a basis $\mathfrak{x}_{1}, \ldots, \mathfrak{x}_{r}$ of $L / K$ (notations of [Sh]). According [Sh], p. 157, (11) there exists a matrix $T=\left\{t_{i j}\right\} \in M_{r}(K)$ such that

$$
\forall k_{1}, k_{2} \in K, i, j=1, \ldots, r \quad \Omega\left(k_{1} * \mathfrak{x}_{i}, k_{2} * \mathfrak{x}_{j}\right)=\operatorname{Tr}_{K / \mathbb{Q}}\left(k_{1} t_{i j} \bar{k}_{2}\right)
$$


([Sh], p. 157, (11)). T has properties

(a) $\bar{T}^{t}=-T$, i.e. $i T$ is hermitian ([Sh], p. $\left.157,(12)\right)$ and

(b) Signature of $i T$ is $(n, r-n)$ ([Sh], p. 160, (25)).

We restrict ourselves by those $A$ whose $T$ (it depends on $\mathfrak{x}_{1}, \ldots, \mathfrak{x}_{r}$ ) satisfy

$$
T=\sqrt{-\Delta} E_{n, r-n}
$$

where $E_{n, r-n}:=\left(\begin{array}{cc}E_{n} & 0 \\ 0 & -E_{r-n}\end{array}\right)$.

2.3. Definitions for the type E. We consider the set of triples $\left(L, H_{L}, \alpha\right)$ where

(1) $L$ is a $K$-vector space of dimension $r$;

(2) $H_{L}$ is a $K$-valued Hermitian form on $L$ of signature $(n, r-n)$ such that there exists a basis of $L$ over $K$ satisfying the condition:

$$
\text { the matrix of } H_{L} \text { in this basis is } E_{n, r-n}
$$

We denote by $H_{L, \mathbb{C}}$ the extension of $H_{L}$ to $L \otimes_{K} \mathbb{C}$.

(3) $\alpha: L \otimes_{K} \mathbb{C} \rightarrow \mathbb{C}^{n}$ is a $\mathbb{C}$-linear map such that $\alpha$ is surjective, and

The restriction of $-H_{L, \mathbb{C}}$ to Ker $\alpha$ is a positive definite form.

Remark. This $\alpha$ is clearly an analog of $\alpha$ of 1.1 . We see that conditions of surjectivity of $\alpha$ hold in both cases, while the property 1.2 apparently has no analog in the number field case.

Theorem 2.6. There is a $1-1$ correspondence between the above $A, \iota, H-$ objects of type (B) (here $A$ is up to isogeny, and $A$ has $T$ satisfying 2.2 ), and the above triples $\left(L, H_{L}, \alpha\right)$ - objects of type $(\mathbf{E})$.

Remark. If the restriction of $\alpha$ on $L \subset L \otimes_{K} \mathbb{C}$ is injective (this holds for almost all triples $\left.\left(L, H_{L}, \alpha\right)\right)$ then the $K$-vector space in $\mathbb{C}^{n}$ mentioned in $(\mathbf{E})$ is $\alpha(L)$.

Proof. $L$ is the same for both types (when we consider $L$ for the type (E), we omit * in $k * l)$. Let the triple $(A, \iota, H)$ of the type $(\mathbf{B})$ be given. There is a canonical decomposition $V=V^{+} \oplus V^{-}$where

$$
\begin{aligned}
& V^{+}:=\{v \in V \mid k * v=k v\} \\
& V^{-}:=\{v \in V \mid k * v=\bar{k} v\}
\end{aligned}
$$

We denote by $\pi_{+}: V \rightarrow V^{+}$the projection along $V^{-}$. Let $\beta: L \rightarrow V$ be the tautological inclusion.

2.9. The triple $\left(L, H_{L}, \alpha\right)$ corresponding to $(A, \iota, H)$ is constructed as follows. We define

$$
\alpha: L \otimes_{K} \mathbb{C} \rightarrow V^{+}=\mathbb{C}^{n}
$$


by the formula $\alpha(l \otimes z)=z \cdot \pi_{+}(\beta(l))$. Formula 2.7 shows that it is well-defined. The form $H_{L}$ is defined by the equality

$$
\forall l_{1}, l_{2} \in L \quad \operatorname{Tr}_{K / \mathbb{Q}}\left(\sqrt{-\Delta} H_{L}\left(l_{1}, l_{2}\right)\right)=\Omega\left(\beta\left(l_{1}\right), \beta\left(l_{2}\right)\right)
$$

Lemma. Formula 2.10 really defines $H_{L}$ uniquely.

Proof. Unicity: we fix $l_{1}, l_{2} \in L$, and we consider a $\mathbb{Q}$-linear form $\gamma: K \rightarrow \mathbb{Q}$ defined by the formula

$$
\gamma(k)=\Omega\left(\beta\left(k * l_{1}\right), \beta\left(l_{2}\right)\right)
$$

There exists the only $k_{0} \in K$ such that $\gamma(k)=\operatorname{Tr}_{K / \mathbb{Q}}\left(k_{0} k\right)$. We see that if 2.10 holds for all pairs $k * l_{1}, l_{2}$, then necessarily $\sqrt{-\Delta} H_{L}\left(l_{1}, l_{2}\right)=k_{0}$.

Existence: Let $\mathfrak{x}_{1}, \ldots, \mathfrak{x}_{r}$ be a basis of $L / K$ such that the corresponding $T$ has the form 2.2. We define $H_{L}$ by the condition that the matrix of $H_{L}$ in $\mathfrak{x}_{1}, \ldots, \mathfrak{x}_{r}$ is $E_{n, r-n} .2 .1$ implies that $H_{L}$ satisfies 2.10 .

To prove 2.5 we recall a well-known

2.11. Coordinate description. The Siegel domain for the present case is the following:

$$
\begin{gathered}
\mathcal{H}_{n, r-n}^{3}:=\left\{z \in M_{n, r-n}(\mathbb{C}) \mid E_{n}-z \bar{z}^{t} \text { is positive hermitian }\right\} \\
\left(\Longleftrightarrow E_{r-n}-\bar{z}^{t} z \text { is positive hermitian }\right)
\end{gathered}
$$

([Sh], p. 162, 2.6). For any $z \in \mathcal{H}_{n, r-n}^{3}$ we can construct an above abelian variety $A_{z}$ (satisfying 2.2) as follows ([Sh]). We fix a basis $e_{1}, \ldots, e_{r}$ of $V$ over $\mathbb{C}$ such that $e_{1}, \ldots, e_{n}$ (resp. $\left.e_{n+1}, \ldots, e_{r}\right)$ is a basis of $V^{+}\left(\right.$resp. $\left.V^{-}\right)$over $\mathbb{C}$. It defines the $K$ -

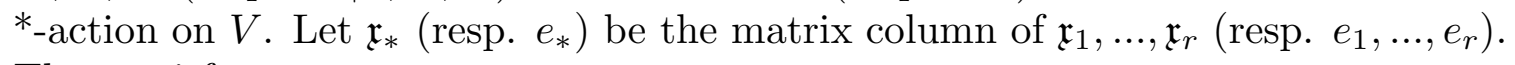
They satisfy

$$
\mathfrak{x}_{*}=Y e_{*} \text { where } Y=\left(\begin{array}{cc}
E_{n} & z \\
\bar{z}^{t} & E_{r-n}
\end{array}\right)
$$

([Sh], p. 162, (35), Type IV). $\Omega$ is defined by 2.1, 2.2. These conditions define $A_{z}$.

Proof of 2.5. 2.13 implies that elements

$$
\lambda_{i}:=\mathfrak{x}_{n+i} \otimes 1-\sum_{k=1}^{n} \mathfrak{x}_{k} \otimes \bar{z}_{k i}
$$

$i=1, \ldots, r-n$, form a basis of Ker $\alpha$. We have

$$
H_{L}\left(\lambda_{i}, \lambda_{j}\right)=\sum_{k=1}^{n} \bar{z}_{k i} z_{k j}-\delta_{i}^{j}=\left\{\bar{z}^{t} z-E_{r-n}\right\}_{i j}
$$

hence 2.12 implies 2.5 .

So, we have constructed a well-defined map from the set of objects of type (B) to the set of objects of type $(\mathbf{E})$. 
To construct the inverse map we need a definition. Let $W$ be a $\mathbb{C}$-vector space. We denote by $\mathfrak{i}(W)$ the complex conjugate space together with a map $\mathfrak{i}: W \rightarrow \mathfrak{i}(W)$ which is an isomorphism of $\mathbb{R}$-vector spaces and satisfies $\mathfrak{i}(z w)=\bar{z} \mathfrak{i}(w), z \in \mathbb{C}$, $w \in W$.

Let a triple $\left(L, H_{L}, \alpha\right)$ be given. Let $(\operatorname{Ker} \alpha)^{\perp} \subset L \otimes_{K} \mathbb{C}$ be the $H_{L, \mathbb{C} \text {-orthogonal }}$ space of Ker $\alpha, \pi_{\alpha}: L \otimes_{K} \mathbb{C} \rightarrow \operatorname{Ker} \alpha$ the projection along $(\operatorname{Ker} \alpha)^{\perp}$, and let us consider the composition $\mathfrak{i} \circ \pi_{\alpha}: L \otimes_{K} \mathbb{C} \rightarrow \mathfrak{i}(\operatorname{Ker} \alpha)$.

We let $V=\mathbb{C}^{n} \oplus \mathfrak{i}(\operatorname{Ker} \alpha)$ (here $\mathbb{C}^{n}$ is the target of $\alpha$ ), and we define the inclusion $\gamma: L \hookrightarrow V$ by the formula

$$
\gamma(l)=\left(\alpha(l), \mathfrak{i} \circ \pi_{\alpha}(l)\right)
$$

The polarization $H$ on $V$ is defined by the same formula (2.10); here $H_{L}$ is given, so $(2.10)$ defines $\Omega$ for any pair $\left(\beta\left(l_{1}\right), \beta\left(l_{2}\right)\right)$. Further, $H$ is defined uniquely by $\Omega$ (but clearly we must prove that this $H$ really is a positive hermitian form). The action of $K$ on $V$ is defined by the property that $k * v=k v$ for $v \in \mathbb{C}^{n}$ and $k * v=\bar{k} v$ for $v \in \mathfrak{i}(\operatorname{Ker} \alpha)$, i.e. $\mathbb{C}^{n}=V^{+}, \mathfrak{i}(\operatorname{Ker} \alpha)=V^{-}$.

Let us prove that the above $V, H, \gamma(L)$ and the action of $K$ really define an abelian variety up to polarization of type (B). Obviously, the signature of the action of $K$ on $V$ is $(n, r-n)$. Further, for $k \in K, l \in L$ we have $\gamma(k l)=k * \gamma(l)$, i.e. $\gamma(L)$ is a $K$-*-lattice. Let $\mathfrak{x}_{1}, \ldots, \mathfrak{x}_{r}$ be a basis of $L$ over $K$ satisfying (2.4). It defines a $n \times(r-n)$-matrix $z=\left\{z_{i j}\right\}$ as follows (this is the same as 2.14):

$$
\alpha\left(\mathfrak{x}_{n+i}\right)=\sum_{k=1}^{n} \bar{z}_{k i} \alpha\left(\mathfrak{x}_{k}\right)
$$

(It is easy to prove that $\alpha\left(\mathfrak{x}_{k}\right), k=1, \ldots, n$ form a basis of $\mathbb{C}^{n}$ ). Condition $(2.5)$ implies that $z \in \mathcal{H}_{n, r-n}^{3}$ (calculations coincide with the ones of 2.15). This means that $\lambda_{i}$ defined by (2.14) with $z_{* *}$ from (2.16) form a basis of Ker $\alpha$. We define

$$
\mu_{i}:=\mathfrak{x}_{i} \otimes 1-\sum_{k=1}^{r-n} \mathfrak{x}_{n+k} \otimes z_{i k}
$$

$i=1, \ldots, n$. Obviously $H_{L, \mathbb{C}}\left(\lambda_{i}, \mu_{j}\right)=0$, this means that $\mu_{1}, \ldots, \mu_{n}$ is a basis of $(\operatorname{Ker} \alpha)^{\perp}$.

Let $Y$ be defined by (2.13) with $z_{* *}$ from (2.16). We have

$$
Y\left(\begin{array}{cc}
E_{n} & -z \\
-\bar{z}^{t} & E_{r-n}
\end{array}\right)=\left(\begin{array}{cc}
E_{n}-z \bar{z}^{t} & 0 \\
0 & -\bar{z}^{t} z+E_{r-n}
\end{array}\right)
$$

hence (2.12) implies that $Y$ is invertible. We define a vector column $e_{*}$ by the formula $e_{*}:=Y^{-1} \cdot \gamma\left(\mathfrak{x}_{*}\right)$. We get immediately using $(2.16),(2.17)$ that $e_{1}, \ldots, e_{n}$ from a basis of $V^{+}, e_{n+1}, \ldots, e_{r}$ from a basis of $V^{-}$. So, results of [Sh] imply that $V, H, \gamma(L)$ and the action of $K$ really define an abelian variety up to polarization of type (B).

The proof of the affirmation that the above two constructions are inverse is practically a tautology. 


\section{Section 3. Exterior powers of abelian varieties with MIQF having $n=1$.}

Let $(A, \iota, H)$ be a triple of Theorem 2.6. The associated triple $\left(L, H_{L}, \alpha\right)$ defines an exact sequence of $\mathbb{C}$-vector spaces

$$
0 \rightarrow \operatorname{Ker} \alpha \stackrel{i}{\hookrightarrow} L \otimes_{K} \mathbb{C} \stackrel{\alpha}{\rightarrow} \mathbb{C}^{n} \rightarrow 0
$$

Let us take the $k$-th exterior power of this exact sequence (Definition 1.4):

$$
0 \rightarrow \lambda^{k}(\operatorname{Ker} \alpha) \stackrel{\lambda^{k}(i)}{\hookrightarrow} \lambda^{k}(L) \otimes_{K} \mathbb{C} \stackrel{\alpha_{k}}{\rightarrow} W_{k} \rightarrow 0
$$

There exists an Hermitian form $\lambda^{k}\left(H_{L}\right)$ on $\lambda^{k}(L)$; recall that

$$
\lambda^{k}\left(H_{L}\right)\left(l_{1} \wedge \ldots \wedge l_{k}, l_{1}^{\prime} \wedge \ldots \wedge l_{k}^{\prime}\right)=\operatorname{det}\left\{H_{L}\left(l_{i} \wedge l_{j}^{\prime}\right)\right\}
$$

It is obvious that if $n=1$ then the restriction of $(-1)^{k} \lambda^{k}\left(H_{L}\right)$ to $\lambda^{k}(\operatorname{Ker} \alpha)$ is positive definite. This means that the triple $\lambda^{k}(L),(-1)^{k-1} \lambda^{k}\left(H_{L}\right), \alpha_{k}$ satisfies conditions of Theorem 2.6 and hence defines an abelian variety (up to isogeny) $\lambda^{k}(A)$ which is called the $k$-th exterior power of $A$. Its signature is $\left(\begin{array}{c}r-1 \\ k-1\end{array}\right),\left(\begin{array}{c}r-1 \\ k\end{array}\right)$.

Remark 3.1. It is easy to see that if $n \neq 1, r-1$ then this construction cannot be applied to abelian varieties of signature $(n, r-n)$; we cannot also define symmetric powers of abelian varieties with MIQF, as well as their tensor products. This is clearly an analog of 1.3 .

Remark 3.2. For the function field case there exists a natural definition of the exterior power of T-motives and 1.7 is a theorem, while for the number field case there is no such definition hence we must consider the above construction - an analog of the Theorem 1.7 — as the definition of the exterior powers of abelian varieties with MIQF.

Remark 3.3. This construction of exterior power can be continued to the corresponding Shimura varieties. Let us recall some definitions of [D]. Attached to a Shimura variety $X$ is an algebraic group $G=G_{X}$ over $\mathbb{Q}$ and a map $h=h_{X}: \mathbb{S} \rightarrow G$ over $\mathbb{R}$, where $\mathbb{S}=\operatorname{Res} \mathbb{C} / \mathbb{R}\left(G_{m}\right)$, satisfying some conditions. A map $\varphi: X \rightarrow Y$ of Shimura varieties comes from a map $G_{\varphi}: G_{X} \rightarrow G_{Y}$ satisfying $G_{\varphi} \circ h_{X}=h_{Y}$. If $X$ is a Shimura variety parametrizing abelian varieties with MIQF of signature $(r, s)$ then $G_{X}=G U(r, s)$. The map $G_{\varphi}: G U(1, r-1) \rightarrow G U\left(\left(\begin{array}{c}r-1 \\ k-1\end{array}\right),\left(\begin{array}{c}r-1 \\ k\end{array}\right)\right)$ corresponding to the above construction is evident. Really, let $L, H_{L}$ be of 2.3 $(n=1)$; then $\gamma \in G U(1, r-1)$ is an endomorphism of $L$ (denoted by $\gamma$ as well) satisfying $H_{L}\left(\gamma\left(l_{1}\right), \gamma\left(l_{2}\right)\right)=\lambda(\gamma) H_{L}\left(l_{1}, l_{2}\right)$. Clearly $G_{\varphi}(\gamma)$ is $\lambda^{k}(\gamma): \lambda^{k}(L) \rightarrow$ $\lambda^{k}(L)$. It is an elementary exercise to check all compatibilities.

\section{References}

[D] Deligne P. Travaux de Shimura. Lect. Notes in Math., 1971, v.244, p. 123 165. Seminaire Bourbaki 1970/71, Exposé 389.

[G] MR1423131 (97i:11062) Goss, David Basic structures of function field arithmetic. Springer-Verlag, Berlin, 1996. xiv+422 pp. 
[GL07] Grishkov, A.; Logachev, D. Duality of Anderson t-motives. https://arxiv.org/pdf/0711.1928.pdf

[L] Logachev D., Congruence relations on Anderson T-motives. In preparation.

[P] Richard Pink, Hodge structures over function fields. Universität Mannheim. Preprint. September 17, 1997

[Sh] Shimura, Goro On analytic families of polarized abelian varieties and automorphic functions. Annals of Math., 1 (1963), vol. 78, p. $149-192$

[W] T. Wedhorn, Congruence relations on some Shimura varieties. J. reine angew. Math., 524 (2000), p. $43-71$

First author: Departamento de Matemática e estatistica Universidade de São Paulo. Rua de Matão 1010, CEP 05508-090, São Paulo, Brasil, and Omsk State University n.A. F.M.Dostoevskit. Pr. Mira 55-A, Omsk 644077, Russia.

Second author: Departamento de Matemática, Universidade Federal do Amazonas, Manaus, Brasil 